\title{
RESPONDIENDO A UN DESAFÍO PENDIENTE EN GEOGRAFÍA: EL ENFOQUE DE GÉNERO VISTO DESDE ESPAÑA*
}

\author{
García Ramón, M. ${ }^{\text {a Dolors }}$ \\ Dpto. de Geografía, Universidad Autónoma de Barcelona, 08193, Bellaterra \\ mariadolors.garcia.ramon@uab.es
}

\begin{abstract}
Resumen: Hace tan sólo quince años que publique un artículo en España en el que yo misma abogaba por la necesidad de no excluir del estudio a la mitad del género humano, y argumentaba que la introducción del enfoque de género era una tarea ineludible en geografía humana. Actualmente se podría asegurar que la consolidación de los estudios de género en la geografia internacional es un hecho muy destacable aunque se observe grandes diferencias de ritmo en este proceso según los diferentes países y regiones del mundo.
\end{abstract}

En primer lugar, en este artículo quiero hacer un balance del desarrollo de las nuevas tendencias a nivel internacional desde la década de los años 1990's y, en segundo lugar, quiero hacer un balance de la producción cientifica en esta temática en España desde la introducción del enfoque.

Palabras clave: geografía y género, geografía feminista, geografía española, postmodernismo, metodologías feministas.

\begin{abstract}
In an article signed by myself just fifteen years ago I was advocating the need of not excluding from our research in human geography half of the humankind, thereby arguing that the introduction of the gender approach was an unavoidable task. Nowdays it could be asserted that gender studies have been fully integrated in international Geography althoug at very different rates in different regions of the world. The present article tries to present first, the recent developments and new trends at the internacional level since the 1990's and, second, to present a survey of the scietific procduction on the field in Spain since the gender approached was introduced.
\end{abstract}

Key words: Gender Geography, feminist Geography, Spanish Geography, postmodersnim, feminist methodologies.

* Recibido: 1-3-05. Aceptado: 5-12-05. 


\section{Introducción}

Hace tan sólo quince años que en un artículo publicado en el Boletín de la Asociación de Geógrafos Españoles yo misma abogaba por la necesidad de no excluir del estudio a la mitad del género humano, y argumentaba que la introducción del enfoque de género era un tarea ineludible en geografía humana (García Ramon, 1989).

No obstante, actualmente se podría asegurar que la consolidación de los estudios de género en la geografía internacional es un hecho muy destacado aunque se observe una gran diferencia en este proceso según los diferentes países y regiones del mundo (Monk, 1996; García Ramon, 2004). En los países anglosajones (que es donde se empezó hace ya unos veinticinco años) su desarrollo ha ido muy lejos, tanto desde una perspectiva teórica como metodológica, pero en los países latinos la "normalización" del enfoque de género en la práctica de nuestra disciplina ha discurrido de forma diferente (Cortesi, 1996; Silva, 2000; Creton, 2002). Hasta hace poco, en este contexto la sociedad y el territorio se consideraban como un conjunto neutro, asexuado y homogéneo; es cierto que con frecuencia se tenían en cuenta las diferencias de clase, pero sin plantear las importantes diferencias que existian entre hombres y mujeres en el uso y vivencias del espacio y del medio.

Pero parece que la situación en el contexto de los países latinos también está cambiando y si bien es cierto que la geografía ha incorporado con cierto retraso este enfoque de género (en comparación con otras ciencias sociales) no lo es menos que su desarrollo y aceptación han sido, en algunos casos, relativamente rápidos. Por ejemplo, es bastante sintomático que el manual de historia de la geografía más conocido Geography and geographers:Angloamerican buman geography since 1945 de R.J. Johnston no incorpora un capítulo dedicado a la geografía feminista hasta la última edición de 2004, de la que es coautor J. Sidaway (quinta edición revisada des de que se publicó inicialmente el libro en 1979). En cambio, en la geografía española, el interesante manual de pensamiento geográfico de Ortega publicado en el 2000 ya incluía un buen número de paginas dedicadas a la reflexión teórica sobre las contribuciones de la geografía feminista. No obstante, cabe señalar que tampoco aquí es la norma y se han publicado recientemente algunos manuales muy interesantes de geografía humana en los que hay escasísimas referencias al enfoque de género (Romero et al. 2004). Pero en la geografía española contamos con el mérito de tener el primer manual de geografía del género en castellano, excelente por cierto (Sabaté et al. 1995).

En este artículo, en primer lugar, quiero hacer un balance del desarrollo de las nuevas tendencias a nivel internacional desde la década de los años 1990's y, en segundo lugar, quiero hacer un balance de la producción científica en esta temática en España desde la introducción del enfoque. Conviene señalar que es importante 
que este balance teórico-metodológico se haga desde una perspectiva latina y mediterránea, pues abundan los estados de la cuestión sobre esta temática en inglés (Bondi, 1990; Pratt 1992, 2004; McDowell 1993; Rose, 1994; Jones III et al., 1997; Johnston \& Sidaway 2004; Panelli, 2004) pero presentan unas insuficiencias muy notorias. Salvo alguna rara excepción (Peake,1989), todos ellos se basan solamente en la investigación que se lleva a cabo en el mundo anglosajón, olvidándose por completo de la realizada en otros ámbitos culturales y linguísticos (García Ramon, 1989; Cortesi, 1996; Fosberg,1990; Sabaté \& Tulla,1992; Baylina \& García Ramon, 2000; Chivallon, 2001). De hecho, se da por supuesto que la investigación que se lleva a cabo sobre la temática de género en el ámbito anglosajón "representa" todo lo que se lleva a cabo hoy en día en la geografía internacional. Pero en geografía el "lugar cuenta" (Monk,1996), y aún más si recordamos que la geografía feminista precisamente ha insistido en que se ha de dar prioridad a las diferencias (WGSG,1997; 2004; Peake \&Valentine, 2003), y que el conocimiento se debe "situar" (Haraway, 1995; Hancock, 2002; Albet et al., 2005).

\section{Nuevas perspectivas teórico-metodológicas}

\subsection{Definiciones}

Se ha definido la geografía del género como la que "examina las formas en que los procesos socioeconómicos, políticos y ambientales crean, reproducen y transforman, no sólo los lugares done vivimos, sino también las relaciones sociales entre los hombres y las mujeres que viven allí y, a la vez, también estudia cómo lás relaciones de género afectan a estos procesos y sus manifestaciones en el espacio y en el medio" (Little et al. 1988: 2). Se debe aclarar aquí que, en principio el término género se refiere a las diferencias originadas social y culturalmente entre lo femenino y lo masculino, mientras que el término sexo se refiere más bien a las diferencias biológicas entre hombre y mujer, aunque últimamente se insiste también en la idea de que el sexo también se construye socialmente (Peake \& Valentine, 2003). Ana Sabaté, en su manual sobre el tema, define la geografía feminista como "aquella que incorpora las aportaciones teóricas del feminismo a la explicación e interpretación de los hechos geográficos" (Sabaté et al., 1995: 16). Se hace necesario explicitar que el mundo anglosajón los términos "geografía feminista" y "geografía del género" son prácticamente intercambiables; en cambio, en los países de tradición latina el término de geografía feminista tiene una connotación más militante (quizás equivocadamente), y el término geografía del género una connotación que parece más aceptada académicamente (no está tan seguroj) aunque es cierto que bajo esta última denominación se 
insiste en la idea de la construcción social del gènero. Yo los utilizaré de forma indistinta en este texto porque creo que se han de reivindicar las aportaciones teóricas de ambos términos.

Lo que es cierto es que la geografía del género ( $y /$ o la geografía feminista) van mucho más allá que la denominada "geografía de las mujeres" en que simplemente se estudiaban las mujeres, pero, en cambio, no se tenía en cuenta la construcción social del género (WGSG, 1984; Bowlby, 1989). Y hoy en día está muy claro que la geografía del género no ha de ser cultivada solamente por las mujeres y estudiar sólo las mujeres (aunque no se niega que no fuera ello necesario en una etapa inicial) sino que en los estudios más innovadores se pone énfasis en que hay que estudiar y comparar los roles de género asignados tanto a hombres como mujeres (Coutras et al. 1989; Paravicini et al., 1990; 2003). Y conviene señalar que no sólo es importante que se incluyan los hombres como objeto de estudio, sino que también es necesario que ellos lleven a cabo investigaciones en esta temática, como afortunadamente ya está sucediendo en los últimos años.

\subsection{El debate postmodernista y la geografía feminista}

En la década de 1990 el postmodernismo, el postcolonialismo y el denominado "giro cultural" están en la base de muchas de las discusiones teóricas en geografía, y también es así en la geografía feminista, sobre todo teniendo en cuenta que ésta ha sido pionera en la introducción del debate postmodernista en la disciplina (WGSG, 1997). De hecho, la geografía feminista y el postmodernismo comparten una visión crítica del pensamiento racionalista y de sus pretensiones totalizadoras y universales pues no cree en la existencia de una conocimiento "real" que sea universal, neutral, objetivo y producto exclusivo de la razón y de la lógica. Así pues, todas las categorías de análisis se ha de "deconstruir" y se han de contextualizar, es decir adaptar a los diferentes lugares y circunstancias. La geografía feminista tiene una larga experiencia (obligada) de "deconstrucción" (Bondi, 1990). Por ejemplo, el concepto de clase social tuvo que adaptarse y combinarse con el de patriarcado en contextos culturales muy diversos, con relaciones patriarcales muy diversas. Las nuevas posiciones teóricas nos invitan a estudiar la complejidad de las experiencias de mujeres (y no de la mujer) y a combinar la dimensión de género con otras causas de la diferencia, como la etnicidad, la clase social, la nacionalidad o la sexualidad (Caballé, 1997; Pratt, 2004). La discusión sobre la diferencia y sobre el significado del lenguaje ha impulsado a la geografía del género a plantearse cuestiones como la representación, la identidad, y el cuerpo temas centrales no sólo de la geografía feminista sino de la geografía postmodernista de principios del siglo XXI (Longhurst, 1997; Longhurst, 2001). 
Se ha insistido en que la categoría de género es tan aplicable a hombres como a mujeres y ello ha llevado al desarrollo de estudios de la masculinidad (Berg \& Longhurst, 2003). Una publicación pionera fue el trabajo de Jackson (1991) sobre la política cultural de la masculinidad donde se estudia, de forma empírica, cómo se construye ésta de forma diferencial en EEUU en la época de la Primera Guerra Mundial y la Inglaterra de la Guerra de las Malvinas (el autor lo hace a través del estudio de la representación de la masculinidad en los carteles de propaganda del ejército). También Massey (1995) y McDowell (1999; 2003) estudian cómo los diferentes lugares, y en particular los de trabajo, incluyen la negociación y la actuación de diferentes identidades de género. En el caso de las industrias de alta tecnología Massey nos recuerda que la masculinidad se construye asociada a interminables horas de trabajo y a la utilización de ordenadores muy potentes, así como el uso de la razón, la "cientificidad" y el pensamiento abstracto. En cambio, McDowell en su estudio sobre chicos de clase trabajadora, muestra que sus identidades masculinas se asocian más al propio trabajo en sí (normalmente en la industria) y al sentido de responsabilidad de ser un posible cabeza de familia más que a todo lo relacionado con la formación en la escuela. Massey ha continuado trabajando sobre las relaciones entre la identidad y los lugares -en particular en ciudades- poniendo un énfasis muy particular en el carácter cambiante y fluido de la identidad.

Bajo la inspiración de teorías fenomenológicas y psicoanalíticas el tema del cuerpo se introdujo relativamente pronto en la geografía del género, al menos si se compara con otros campos de la geografía (Rose, 1995). Se critica un discurso geográfico que da por descontado que el cuerpo está ahí, discurso que está sostenido por una separación cartesiana entre la mente y el cuerpo. También se discuten los costes que comporta este olvido de lo que en geografía se ha llamado la escala corporal y se considera que la experiencia del cuerpo es esencial para comprender las relaciones de las personas con los entornos físicos y sociales (McDowell, 1995; 1999; Longhurst, 1997; Johnston, 1996). Los estudios en que se incluye el cuerpo como categoría de análisis han proliferado en geografía feminista (y también en otros campos de la geografía) y pretenden provocar nuevas formas de entender el poder, el conocimiento y las relaciones sociales entre la gente y los lugares (Longhurst, 2001; Bru, 2004).

La sexualidad es otro tema que se ha introducido más recientemente -y que ha tenido también cierta repercusión en medios feministas, aunque todavía se debate si los estudios de la sexualidad se han de incluir o no en la geografía feminista (Domosh, 1999; Pratt, 2004). Desde la década de 1990's ya se empezó a estudiar cómo el espacio y la sexualidad se constituyen mutuamente (Bell et al. 1995). En particular se estudiaron los espacios homosexuales de la ciudad y su relación con el proceso de elitización de barrios urbanos (Knopp, 1990;García-Escalona, 2000; Santos, 2002); pero también se estudiaron otros lugares y otras sexualidades disidentes analizándose, por ejemplo, la construcción de la identidad lesbiana en el mundo rural o en los desfiles urbanos (Little, 2002; Johnston 2001) (pero cabe señalar que los estu- 
dios sobre espacios lesbianos son más bien escasos). El impacto de la teoría "queer" (teoría de lo transexual) ha sido importante en los estudios de la geografía de la sexualidad y ésta plantea que la identidad sexual no es nunca fija sino que siempre está en proceso de cambio (Hubbard 2000; Kitchin et al. 2003). Según estos trabajos, la sexualidad se construye a través de procesos materiales y culturales como una categoría social fluida más que rígida, inspirándose en los trabajos de J. Butler, que nos señala que el concepto de sexo es también un construcción social (igual que el del género). Los trabajos en esta línea versan sobre lugares y temas diferentes desde el estudio de los espacios cotidianos (Kitchin et al. 2003) al de los desfiles "gay" (u homosexuales) y de su impacto en la construcción de estos espacios y el desarrollo del turismo urbano (Johnston, 2001; 2004).

Señalaremos cuatro manuales que pueden ser representativos de este periodo y estos enfoques. Uno de ellos es de Nelson y Seager, A companion to Feminist geography (2005). Feminism and Geograpby de Rose (1994) nos aporta una reflexión teórica muy sólida, no sólo sobre la geografía del género sino que va más allá; se trata de una reflexión crítica sobre el género de la geografía. La autora critica la masculinidad de la mirada del geógrafo/a y estudia la penetración sutil pero intensa de las epistemologías masculinas a lo largo de la historia de la disciplina. Otro importante manual postmodemo es el publicado por el WGSG (autor del conocido primer manual de 1984) que podríamos considerar el primer manual postmoderno. No se habla ya de geografía feminista sino de "geografías feministas" aludiendo a la diversidad y diferencias que ya se observan en la literatura geográfica feminista (pero curiosamente no incluyen los trabajos de geógrafas feministas no anglófonas, ni incluso los trabajos publicados en inglésj). Quiero señalar con especial interés el enfoque del manual de G. Valentine, Social Geographies: Space and Society (2001) en cuyo título e índice no aparecen en absoluto la palabras género, mujer, feminismo etc. pero cuyos contenidos son el resultado de un analisis feminista de la sociedad y del medio. Es de celebrar que la autora haya sido capaz de llevar a cabo tal cometido con éxito pues facilita la integración del enfoque del género en diversos campos de la geografía.

El mismo grupo de la WGSG acaba de publicar un manual mucho más modesto y en formato CD (WCSG, 2004) con ocasión de la celebración de los veinte años de la publicación del primer manual en el marco del 30 Congreso de la Unión Geográfica Internacional (UGI) celebrado en Glasgow en agosto de 2004 (la innovación más significativa es la inclusión de las geografía emocionales). En este congreso mencionado, se ha de señalar el papel de la Comisión de Género y Geografía de la Unión Geográfica Internacional que organizó un número sustantivo de sesiones sobre género, tal como ha ido realizando desde su creación en 1988 en el Congreso de la UGl en Sydney (aunque hay que decir que en este congreso también en otras sesiones se presentaron un buen número de comunicaciones sobre género, lo que prueba la buena salud y a la vez la madurez del enfoque (UGI, 2004). La Comisión ha sido (y es) un motor importantísimo para el desarrollo de los estudios de género en la geo- 
grafía internacional y sus casi 400 miembros se reparten entre más de 58 países. Las actividades de la Comisión realmente representan un esfuerzo exitoso al situar el conocimiento geográfico dentro de una geografía del género inclusiva y "situada", es decir realmente internacional.

Es de destacar que durante esta etapa (en 1994) se inició la primera revista de geografía feminista, Gender, Place and Culture que ya anunciaba en su primer editorial que no quería encerrar a la geografía feminista en un "ghetto" pues este enfoque ya se había afianzado en muchas otras revistas de prestigio (Bondi \& Monosh, 1994). La revista ha sido un foro importantísimo de debate teórico muy respetado por amplios sectores de la geografía. Las aportaciones interdisciplinarias han sido muy interesantes y es cierto que la revista no ha contribuido a encerrar a la geografía feminista en un "ghetto", pero es verdad que se observa en ella un sesgo anglosajón (no sólo por la lengua sino también por los contenidos). En una reciente editorial se hace una autocrítica en este sentido y se anuncian medidas para corregir dicho sesgo (Peake \& Valentine, 2003). Hay que celebrarlo, ya que no hay peor práctica que la de ignorar la propia "situación" o posicionalidad o darla por universal.

\section{3 ¿Hacia unas metodologías feministas?}

Hay que hacer hincapié en que el giro cultural y el postmodernismo ha reactivado el debate metodológico en la geografía en general y, muy en particular, en la geografía feminista. Con cierta frecuencia se ha afirmado en círculos feministas que la investigación racional, cuantitativa y objetiva está ligada a la masculinidad, y que la "blanda", cualitativa y emocional está más bien asociada con la feminidad (Professional Geographer, 1994; 1995; 2001). Pero es opinión muy extendida que es necesario dejar de lado estas concepciones dualistas (y de adscripción rígida a los géneros) y que se deben crear propuestas epistemológicas que no sean oposicionales, porque lo único que es importante en la elección del método es el rigor de análisis y los objetivos de estudio (McDowell, 1992; Baylina, 1997). Se podría afirmar que en la geografía del género actual no existe un método feminista de análisis, aunque sí es cierto que en la práctica se observa un sesgo claro hacia los métodos cualitativos e intensivos (Prats 1998; Baylina 2004). También es cierto que la geografía feminista ha sido pionera en el re-descubrimiento reciente en la disciplina de los métodos cualitativos, hecho que en otras ciencias sociales ya se había producido con anterioridad (García Ballesteros,1998; Pedone 2000; Crang, 2002).

Varios temas han sido de intenso debate, entre ellos cabe señalar el tema de la representación y el de la reflexividad. ¿Quién se otorga el poder de hablar en nombre de quién, quiénes? es un problema que se agudiza en estudios sobre el Tercer Mundo llevados a cabo por occidentales (Townsend, 1995; Miraftab, 2004). La diná- 
mica de poder entre el sujeto investigador y el sujeto investigado ha sido tema de discusión desde hace tiempo (Katz, 1994); se reconoce que todo conocimiento es situado o posicionado (England, 1994; Rose, 1997) y se rechaza la imagen de un sujeto investigador como experto/a omnipotente que controla al sujeto investigado y el proceso de investigación. La metodología feminista reconoce explícitamente que los resultados de la investigación no son neutros sino que están influenciados por esta posicionalidad y en todo proceso de investigación se hace necesaria la reflexividad (es decir la introspección autocrítica y comprensiva y un análisis profundo de la propia identidad como sujeto investigador) (England 1994; Valentine, 2002).

Quizás conviene señalar que en la actualidad el sesgo observable a favor de los métodos cualitativos se debe a diferentes factores. Por un lado es cierto que los métodos cuantitativos se han adscrito a un modelo positivista de ciencia cuyos principios (neutralidad y objetividad) son difícilmente conciliables con el proyecto feminista. Por otro lado, los datos estadísticos (que son los que, en general, están en la base de estos métodos cuantitativos) con frecuencia no se desagregan por sexo o no ofrecen los matices necesarios para un análisis descle la perspectiva de género. Por ejemplo, de momento, muchos censos sólo dan información sobre el trabajo remunerado y no sobre el denominado "invisible", mayoritariamente llevado a cabo por mujeres. Además los métodos intensivos o cualitativos ofrecen la posibilidad de estudiar procesos sociales poco estandarizados y trabajados, como es el caso de muchos de los temas estudiados desde la perspectiva del género (Crang, 2003). Pero tal como ya se ha mencionado, un número creciente de investigadores/as argumentan que hay que superar esta dicotomía metodológica. Su desaparición conducirá al desarrollo de estrategias de investigación que reconozcan la complementariedad de algunas técnicas cualitativas y cuantitativas. Muy en particular, en los dos últimos años se ha hecho referencia a los Sistemas de información Geográfica (Kwan, 2002) y a la posibilidad de que los SIG se planteen y utilicen de una forma más reflexiva, más contestataria y más congenial con las epistemologías feministas. No deja de ser significativo que la revista Gender, Place and Culture haya dedicado recientemente todo un dossier al tema. Los artículos analizan las causas del desencuentro entre los SIG, la geografía crítica y la geografía feminista, y se intenta establecer puentes entre las diferentes posiciones. Todo parece indicar que esta andadura ya ha empezado (McLafferty, 2005)

\section{Balance de la producción científica española en las dos últimas décadas}

Voy a plantear un balance de la investigación en la geografía española en esta temática, y de buen principio, quiero remarcar que no es un trabajo exhaustivo sino 
que pretende solamente subrayar algunas cuestiones que me parecen fundamentales. El balance lo plantearé en base a cuatro ejes temáticos, a saber: género y mujer en la geografía académica, el análisis de los espacios rurales, el estudio del medio urbano y, finalmente, la aportación de las viajeras a la geografía postcolonial. Los dos temas centrales (estudios rurales y estudios urbanos) son quizás los que han acaparado más investigaciones y por ello les dedico algo más de espacio. No obstante, quiero señalar que dedico bastante atención al último tema, ya que los estudios de viajeras están en la base de enfoques culturales muy recientes a nivel internacional y en el panorama de la geografía española resultan muy novedosos. Los temas de población y de familia se han integrado en los apartados centrales sobre el mundo rural y urbano.

\subsection{Género y mujeres en la geografía académica}

Inicialmente se realizaron varios trabajos que estudiaban la presencia y estatus de las mujeres en la geografía española así como su producción científica, a través del análisis de publicaciones en revistas especializadas (García Ballesteros, 1982; Castañer et al,1985; García-Ramón, 1988). La presencia numérica era relativamente alta en comparación con otras disciplinas más establecidas, en parte debido a que la licenciatura de geografía no se creó hasta finales los años 70 y estaba bastante enfocada a la enseñanza. Sin embargo, el estatus, el poder y la producción científica de las mujeres en la disciplina era más bien bajo. Recientemente, se ha vuelto a estudiar el tema y se comprueba que, aunque por una parte se ha elevado el status académico de las mujeres geógrafas, por otra se observa que la disciplina está experimentando un proceso de masculinización tanto del profesorado como del alumnado (Pujol, et al. 2003; García Ramon, 2005). Ello es probablemente debido, a la profesionalización y tecnificación de nuestra disciplina y al hecho conocido de que las mujeres tienen menos tendencia a escoger carreras y profesiones técnicas y científicas.

También se estudió la repercusión internacional de este enfoque a través del estudio bibliométrico de las publicaciones en 75 revistas de geografía (en 23 países), analizando la evolución de las diferentes temáticas (Caballé, 1997) Se detecta que a pesar de la gran influencia que en sus origenes tuvo la geografía anglosajona, actualmente están surgiendo diferentes modelos territoriales para llevar a la práctica este enfoque. Es decir, el contexto regional ha adaptado las influencias anglosajonas originarias y ha hecho aparecer diferentes estilos académicos y diferentes repertorios temáticos que se pueden "situar" en los cuatro grandes agrupamientos regionales utilizados en el análisis (revistas en inglés, revistas en francés y del área mediterránea, revistas centroeuropeas y escandinavas, y revistas del Tercer Mundo) (García Ramón et al. 1999). 


\subsection{Los espacios rurales}

Durante mucho tiempo, esta línea de estudios ha sido la más importante en consonancia con la tradición ruralista de la geografía española. Las investigaciones las han llevado a cabo, en gran parte, los dos grupos de estudios de género que surgieron a finales de los 80 en la Universidad Complutense de Madrid (dirigido por Ana Sabaté) y en la Autónoma de Barcelona (dirigido por M. Dolors García Ramón), grupos que sin duda alguna han sido el eje animador y aglutinador de los estudios de género en la geografía española. Se desarrollaron fundamentalmente dos líneas de investigación, referidas ambas al trabajo de las mujeres en el ámbito rural: la contribución laboral de las mujeres en las explotaciones agrarias familiares y el papel que desempeñan en la diversificación económica de las zonas rurales (Sabaté et al. 1992).

Dentro de la primera línea cabe señalar una serie de estudios sobre la contribución de la mujer en las explotaciones familiares agrarias de diferentes espacios regionales. Se constató que su aportación de trabajo es muy significativa cuando se contabiliza todo el trabajo real (productivo y reproductivo). El trabajo de la mujer en la explotación agraria es discontinuo, irregular y muy diversificado y se constata que una buena parte de las tareas productivas son difíciles de separar del trabajo doméstico y por lo tanto son difíciles de contabilizar y se recogen mal en las estadísticas agrarias. En definitiva, se demostró que la supervivencia de la explotación familiar agraria en España está condicionada a la participación de la mujer, tanto en áreas donde la actividad agrícola es más bien marginal como en áreas de agricultura intensiva y competitiva (García-Ramón et al. 1995). También la investigación contribuyó al debate teórico en torno a la división entre el trabajo productivo y reproductivo y quedó claro que cualquier intento de explicar el trabajo de las mujeres en este contexto necesita considerar el control patriarcal del proceso de trabajo y la propiedad de los medios de producción (Solsona, 1989). Asimismo, aportó ideas significativas sobre la construcción cultural de la masculinidad y la feminidad en el contexto rural en diferentes Comunidades Autónomas (Baylina et al, 2000).

Se estudió también el rol de las mujeres en el proceso de reestructuración rural y ello ha resultado muy útil para el diseño de políticas rurales por parte de la administración. Unos primeros trabajos se centraron en los procesos de diversificación económica, sobre todo en la localización de industrias ligeras, y se puso de manifiesto que las ventajas que ofrecían las mujeres como mano de obra barata y no conflictiva eran un factor muy importante de atracción (Sabaté, 1993). Más adelante, se estudiaron en diversas Comunidades Autónomas otro tipo de actividades, algunas innovadoras como el turismo rural (Cánoves et al., 1997) o el teletrabajo, y algunas "tradicionales" como el trabajo en agroindustrias o en el propio domicilio (García-Ramón et al. 2000). Para diversas Comunidades Autónomas, se ha estudiado, con un enfoque integrador muy interesante y en base a metodos de análisi intensivos, el trabajo 
informal industrial a domicilio (Baylina et al. 1998). Es cierto que los trabajos desarrollados por estas mujeres se caracterizan por situarse próximos a los circuitos sumergidos o claramente irregulares, por ser escasamente exigentes en cualificación laboral (salvo la aplicación de las habilidades femeninas adquiridas en la propia distribución de roles domésticos), por tratarse de actividades apenas mecanizadas, por su fuerte exigencia de mano de obra, con remuneraciones bajas, y además con carácter preferentemente estacional. Pero también es cierto que las mujeres contituyen un capital social y humano muy importante para el desarrollo rural y local (Prados, 2000; Pallarés et al. 2004; Nieto, 2004), y como investigadoras comprometidas con la igualdad de oportunidades no debemos marginar su rol (aunque sea de este tipo). Al contrario debemos hacerlo visible y denunciar las circunstancias específicas en que se desarrolla su trabajo e insistir en la necesidad de un cambio sustancial en los roles y relaciones de género (Sabaté, 2002).

Recientemente se han abordado dos temáticas que se encuadran en el marco teórico de las relaciones género/medioambiente y de la construcción de nuevas identidades rurales (Sabaté, 2000). Por una parte, se ha trabajado en la agricultura ecológica, es decir en la participación de las mujeres en las distintas fases productivas de los alimentos biológicos, tratando de responder a la cuestión de si hombres y mujeres tienen una relación diferente con dicha producción (López, 2000). Los resultados vienen a demostrar que las mujeres productoras están en situación de rentabilizar plenamente sus ventajas y situarse con protagonismo en un sector emergente en las zonas rurales. Por otra parte, se está trabajando en el estudio del papel de las mujeres rurales en la gestión de los recursos naturales y en el desarrollo sostenible, en particular, en zonas con espacios protegidos (Canosa et al. 2000). Se hace hincapié en las consecuencias que para las mujeres han tenido las actuales políticas de desarrollo rural y de conservación de la naturaleza implementadas desde las diferentes administraciones públicas, tanto de España como de la Unión Europea.

\subsection{Los espacios urbanos}

Los primeros trabajos se centraron en los espacios cotidianos (García Ballesteros, 1986) y en la movilidad femenina, sobre todo en relación con el viaje al trabajo, tema de estudio muy arraigado en la geografía (Díaz et al., 1989). Pero ya a principios de los noventa se emprendió el estudio de los tiempos de las mujeres en la ciudad, inspirándose en los debates de la izquierda italiana (Prats et al. 1995; Prats et al., 2004), siendo los primeros trabajos publicados en castellano sobre el tema. Ante el reto de la incorporación masiva de las mujeres a la vida productiva y la rigidez de los estilos de vida y los horarios de la ciudad se pretendía suministrar elementos para el conocimiento del uso del tiempo por parte de las mujeres, estudiar los horarios de comercios y servicios y su adecuación a la demanda, y emitir recomendaciones orientadas 
a favorecer una mayor libertad e igualdad en el uso del tiempo. Se constató para Barcelona que los diversos tiempos (el del trabajo productivo y reproductivo, el de ocio y el nocturno) competían tremendamente, pero el tiempo del trabajo productivo emergía claramente como el tiempo central, el más rígido, alrededor del cual se organizan los demás, lo que crea sensación de angustia y de "hambre de tiempo". A partir de las recomendaciones, el Ayuntamiento de Barcelona inició una serie de políticas que proponían, a título experimental, una serie de cambios en los horarios de servicios, equipamientos y comercios en un barrio de Barcelona. El tema de la flexibilización de los horarios de trabajo también fue central para el estudio del trabajo de la mujer en el sector de comercio al detalle en áreas metropolitanas; no obstante, en este caso, se trata de una flexibilidad que no beneficia a las mujeres sino tan sólo a las empresas (Ortiz et al. 2000). Tema importante tarnbién ha sido el de las nueva formas de familia y cómo incide en las relaciones de género a nivel español e internacional. (Solsona et al, 1995; 2005).

El papel de las mujeres en la defensa del medio ambiente urbano también se ha abordado en un estudio sobre movilizaciones ciudadanas-lideradas por mujeres (Bru,1995; 1996). Se trata de un estudio de carácter comparativo regional sobre movilizaciones frente a riesgos ambientales (relacionados con la presencia de residuos industriales) en municipios de diversas Comunidades Autónomas. Se constata que las mujeres entienden el medio ambiente de forma bastante alejada al concepto rígido y androcéntrico que se acepta normalmente. Y se plantea la necesidad de definir este concepto de forma más amplia, de modo que sea capaz de englobar una percepción mucho más ligada a las consecuencias de los impactos ambientales en la vida cotidiana (en la salud, en el entorno, en el bienestar de la familia etc.).

Otra línea de investigación reciente ha sido el análisis de una serie de operaciones urbanas, llevadas a cabo en los últimos quince años en ciudades catalanas de distintos tamaños (Ortiz et al. 2002). Es un hecho que las mujeres han estado ausentes de la planificación urbana, no sólo como usuarias del espacio público sino tambien como urbanistas Así pues, se han estudiado una serie de acciones de rehabilitación de espacios urbanos que integraban en sus objetivos la lucha contra la exclusión socioespacial, analizando por una parte los conceptos y estrategias que subyacían en estas actuaciones y por otra el carácter y grado del impacto real de tales medidas sobre la exclusión social y de género. Se constata el potencial integrador de los espacios públicos y se demuestra que su diseño es un elemento crucial para fomentar la presencia de las mujeres, para la creación de ámbitos social y culturalmente significativos para las mujeres, y en definitiva para fomentar los procesos de emancipación (Ortiz, 2004). Y finalmente, quisiera destacar un tema novedoso para la geografía española y que tiene potencialidad de futuro, el de los espacios lesbianos y gays en las ciudades (García Escalona, 2000; Santos, 2002). En estas recientes investigaciones no sólo se destacan los procesos de gentrificación que se originan sino que se 
observa cómo la apropiación de estos espacios urbanos forma parte de una estrategia de empoderamiento de estos grupos.

\subsection{Los estudios poscoloniales y la contribución de las viajeras}

Los estudios postcoloniales agrupan a aquellos trabajos que critican los desiguales procesos de representación con los que la experiencia histórica del Tercen Mundo antes colonizado llega a conceptualizarse en Occidente (García Ramón \& Nogué, 1999). Este marco conceptual empieza a ser corriente en los estudios culturales del mundo anglosajón y el mismo ha servido para realizar una revisión crítica de la historia de nuestra disciplina, frecuentemente a través del estudio de los libros de viaje (Riudor, 2005; Nogue et al. 2005). En esta línea está claro que los plantemamientos de E. Said en 1978 sobre el orientalismo son una aportación crucial a este marco de análisis, aunque los recientes estudios postcoloniales feministas critican sus planteamientos por ser totalizadores (la heterogeneidad del poder colonial queda muy difuminada) y sobre todo porque pone el acento en los aspectos masculinos del proceso colonial (el dominio, el control y las estructuras de poder), reforzando la visión tradicional de que la mujer no había jugado ningún papel destacable en el proceso colonizador. De hecho se ha mostrado que el estudio crítico de los relatos de viajeras del siglo XIX y principios del XX tienen un gran potencial para cuestionar algunos aspectos de las teorías más establecidas sobre el colonialismo y sobre la historia de nuestra disciplina (Domosh, 1991; Nogué 2005). Ahora se intenta evaluar la contribución de la mujer blanca y occidental tanto al proceso como al discurso colonial, analizando por una parte su complicidad (y, a su vez, su resistencia al proyecto colonial) y destacando, por otra, su papel como agente cultural en la formación de las relaciones imperiales (Albet et al. 1999).

Esta ambigüedad confería, con frecuencia, a las mujeres una gran libertad de acción y de palabra respecto al proyecto colonial y es más frecuente encontrar críticas al mismo en los relatos de mujeres que en los de varones. El caso de Isabelle Eberhard en Argelia es un buen ejemplo de esta compleja relación entre resistencia y complicidad que las mujeres experimentaron en relación con el proyecto colonial (García Ramón et al. 1998). Isabelle (nacida en Ginebra pero de familia rusa) durante la mayor parte de su estancia en Argelia fue considerada como una "enemiga de Francia" por sus actitudes, sus escritos y contactos con la gente local, pero al final de su vida fue astutamente utilizada por el general Lyautey para recopilar información sobre las tribus nómadas del Sur argelino. No obstante, el discurso de Eberhardt difumina las fronteras entre los estereotipos del colonizador y colonizado, y es una clara desviación con respecto al discurso orientalista establecido 
Esta ambivalencia sobre el proyecto colonial es casi paradigmática en el caso de la catalana Aurora Bertrana en su libro de 1935 sobre Marruecos (Nogué et al., 1996; García Ramón et al. 1998; Albet et al. 2003). En realidad, Bertrana no puede oponerse a la acción colonizadora ("civilizadora") de una Europa cuya cultura admira, y ésta es, en el fondo, su valoración del Protectorado Francés en Marruecos a pesar de sus retóricas condenas de principio. A la vez, su convicción de que España no está suficientemente "civilizada", esto es europeizada, como para poder colonizar, la lleva a distanciarse de la acción colonial española en el Protectorado de Marruecos. Y en este punto, su vinculación con el ideario catalanista esté probablemente en la raíz de su postura. Pero también su condición de mujer le facilita el distanciamiento de la colonización española, cuyos agentes son hombres, funcionarios o militares con los que no siente afinidad ninguna.

Pero la combinación de las experiencias de raza, nacionalidad, género y clase social hace que el panorama sea mucho más complejo y no se pueda afirmar rotundamente que las viajeras o exploradoras, por su condición de mujer, tengan una actitud menos racista o más crítica con el proyecto colonial. Por ejemplo, la británica Gertrude Bellg- gran conocedora de Oriente Medio y colega de Lawrence de Arabiatrabajó para el Imperio Britànico y fue una pieza clave en la creación y el diseño de las fronteras de Irak en 1921 (en particular las del Sur) (García Ramón, 2003). Sin embargo, aunque en su porte era inequivocamente imperial, al mismo tiempo se las arregló para establecer una cercanía personal con muchos de los árabes con quienes trabajó, y se hizo propagandista entusiasta de su cultura e historia pasada. En estos aspectos de comportamiento y actitud podemos ver y leer en sus textos una opinión diferente en términos que generalmente están ausentes en informes "más objetivos" de funcionarios coloniales, preocupados por su carrera administrativa.

\section{A modo de conclusión}

A principios del siglo XXI se puede afirmar que la consolidación de los estudios de género internacional en la geografia es un hecho muy destacable, aunque se observe grandes diferencias de ritmo en este proceso según los países y regiones del mundo. Y tal como hemos analizado en la primera parte del artículo, el enfoque de género y feminista ha planteado a la geografía un estimulante desafío intelectual y conceptual. Cabe señalar que si finalmente las mujeres son visibles en el paisaje geográfico, es debido tanto a los cambios que se han dado dentro de la geografía misma como de los cambios ocurridos en nuestro entorno social. La geografía, al igual que cualquier otra disciplina social, es un producto de su tiempo y todo conocimiento es una construcción social y como tal refleja las condiciones bajo las que éste se produce y se transmite. 
Y en España, podríamos afirmar que el enfoque de género ha contribuido significativamente a la investigación geográfica, en particular en los campos de la geografía cultural y social. Me parece que ha contribuido a problematizar algunas temáticas de la geografía que la vocación pragmática de una geografía aplicada, muy en auge en nuestro país, tiende con frecuencia a pasar por alto. Además, el empleo de técnicas y métodos de análisis potentes y presuntamente neutrales -que caracteriza a una buena parte de la geografía española moderna- ignora, en efecto, dimensiones fundamentales de la realidad cultural y social, entre ellas las relaciones de género que impregnan y condicionan tantos aspectos de la vida coridiana, de modo particular la concepción misma del trabajo y el uso y vivencias del espacio y del medio.

\section{Referencias bibliográficas}

Albet, Abel \& García Ramón, M. Dolors (1999): "Reinterpretando el discurso colonial y la historia de la geografía en una perspectiva de género" IN Nogué, Joan \& Villanueva, José Luis (eds) España en Marruecos: Discursos geográficos e intervención territorial, Lleida: Milenio, pp. 35-54.

Albet, Abel \& García Ramón, M. Dolors (2003): "Discours sur l'Orient et colonialisme: narratives de voyages et regards féminins chez Aurora Bertrana", Agora: revue d'études litteraires, 5, pp. 46-57.

Albet, Abel, Benach, Núria \& Clua, Anna (2005): "Conocimientos situados": reflexión sobre las geografías de la Geografia (Crónica de una viaje al 100 Congreso de la Association of American Geographers", Documents d'Anàlisi Geogràfica, 45 (en prensa).

Baylina, Mireia \& García Ramón, M. Dolors (1998): "Homeworking in rural Spain: a gender approach", European Urban and Regional Studies, 5(1), pp. 55-64.

Baylina, Mireia \& García Ramón, M. Dolors (2000): "Estudios rurales y género en la geografía europea", Actas del X Coloquio de Geografia Rural en España, Lleida: Universidad de Lleida, pp. 467-479.
Baylina, Mireia (2004): "Metodología para el estudio de las mujeres y la sociedad rural", Estudios Geográficos, 65 (254), pp. 5-28.

Bell, David and Valentine, Gill (1995) (ed): Mapping desire: geographies of sexuality, New York: Routledge.

Berg, Lawrence \& Longhurst, Robyn (2003): "Placing masculinities and geography", Gender, Place and Culture, 10(4), pp. 351-360.

Bondi, Liz (1990): "Progress in geography and gender: feminism and difference", Progress in Human Geography, 14(3), pp. 428-436.

Bondi, Liz and Domosh, Mona (1994): "Editorial", Gender, Place and Culture, 1, pp. 3-5.

Bowlby, Sophie. (1989): "Geografía feminista en Gran Bretaña: una década de cambio", Documents d'Anàlisi Geogräfica, 14, pp. 15-29.

Bru, Josepa (1995): "El medi está androcentrat. Qui el desandrocentritzarà? Experiència femenina, coneixement ecològic $\mathrm{i}$ canvi cultural", Documents d'Anàlisi Geogrăfica, 26, pp. 271-276. 
Bru, Josepa (1996): "Spanish women against industrial waste: a gender perspective on environmental grasroots movements" en Rocheleau, D. et al.(eds.) Feminist Political Ecology, Londres: Routledge, pp. 105-124.

Bru, Josepa (2004): "Entre el cos i l'ànima: dona, natura i els paisatges de la captivitat", Els paisatges de la posmodernitat.II Seminari Internacional sobre Paisatge, Olot, Novembre (en prensa).

Caballé, Alba (1997): "Aproximación al marco teórico y metodológico en la investigación de geografía del género", Cuadernos Geográficos, 27, pp. 7-27.

Canosa, Elisa et al. (2000): La mujer y la conservación de la naturaleza en España: su papel en la gestión de los recursos naturales $y$ en el desarrollo rural sostenible. Proyecto del Instituto de la Mujer, Madrid.

Cànoves, Gemma (1995): "Estructura familiar i treball de la dona a l'agricultura: el cas d'Osona i el Baix Empordà", Documents d'Anàlisi Geogràfica, 26, pp. 53-71.

Cànoves, Gemma \& Villarino, Montserrat, (1997): "El turismo rural en Cataluña y Galicia, una alternativa o complemento a la explotación familiar:Las mujeres sus nuevas protagonistas" en Valenzuela, $M$. (ed.) Los turismos de interior. El retorno de la tradición viajera, Madrid: Universidad Autónoma de Madrid, pp. 353-368.

Castañer, Margarida \& Centelles, Núria (1985): "La mujer y la geografía universitaria espanola", Documents d'Anàlisi Geogrà-fica, 14, pp. $73-88$

Chivallon, Claude (2001): "Les géographies féministes. Un plaidoyer convaincant pour la constitution de connaissances 'situés'" IN Staszak, Jean F.(dir) Géographies anglo-saxonnes. Tendances contemporaines, París: Belin, pp. 57-62.

Cortesi, Gisela (1996): "Si può parlare di una geografia del genere in Italia? IN. Gisela.
Cortesi and M. Luisa Gentileschi, eds.: Donne e geografia:studi, richerche, problemi. Milano: Franco Angeli, pp. 25-41.

Coutras, Jacqueline.\& Fagnani, Jeanne. (1989): Sexes et éspace, Espace, Populations et Societé, 1, pp. 11-14.

Crang, Michael (2002): "Qualitative methods :the new ortodoxy?", Progress in Human Geography, 26(5), pp. 647-655.

Crang, Michael (2003): "Qualitative methods: touchy, feely, look-see? Progress in Human Geography, 27(4), pp. 494-504.

Creton, Dominique (2002): "Editorial", Espace, Populations, Societés, 3, pp. 253255.

Domosh, Mona (1991): "Towards a feminist historiography of geography" Transactions of the Institute of British Geography, 16(1) pp. 95-104.

Domosh, Mona (1999): "Sexing feminist geography", Progress in Human Geograpby" (23(3), pp. 429-436.

England, Kim (1994): "Getting personal: reflexivity, positionality and feminist research", Professional Geographer, 46(1), pp. 80-89.

Fenster, Tovi (2003): "Espais separats-Espais compartits al Jerusalem d'avui" Treballs de la Societat Catalana de Geografia, 54, pp. 61-78.

Fosberg, Gunner (1990): "On feminism and geography in Sweden", Nordisk Samballsgeografisk Tidskrift, 11, pp. 186-211.

García Ballesteros, Aurora (1982): "El papel de la mujer en el desarrollo de la geografia" en Duran, M.A. (ed.) Liberación y tupopia. Madrid: Akal, pp. 119-141.

García Ballesteros, Aurora (ed) (1986): El uso del espacio cotidiano, Publicaciones de la Universidad Autónoma de Madrid.

García Ballesteros, Aurora (1998) (coord.): Métodos y técnicas cualitativas en geografía social, Barcelona: Oikos-Tau. 
García-Escalona, Emilia (2000): "Del armario al barrio: aproximación a un nuevo espacio urbano", Anales de Geografía de la Universidad Complutense, 20, p. 437.449.

García Ramón, M. Dolors; Castañer, Margarida \& Cetelles, Núria (1988): "Women and Geography in Spanish Universities", Professional Geographer, 40(3), pp. 307315.

García Ramón, M. Dolors, (1989): "Para no excluir del estudio a la mitad del género humano: un desafío pendiente en geografía humana", Boletín de la Asociación de Geógrafos Españoles, 9, pp. 27-48.

García Ramón, M. Dolors; Villarino, Montserrat; Baylina, Mireia \& Canoves, Gemma (1993): "Farm women, gender relations and household strategies in the coast of Galicia", Geoforum, 24(1), pp. 517.

García Ramón, M. Dolors; Cruz, Josefina; Salamaña, Isabel; Villarino, Montserrat, (1995): Mujer y agricultura en España. Género, trabajo y contexto regional, Barcelona: Oikos-Tau.

García Ramón, M. Dolors, Albet, Abel (1998a): "Los relatos de mujeres viajeras ¿Una mirada crítica sobre el colonialismo? Isabelle Eberhard (1877-1904)", Finisterra. Revista Portuguesa de Geografia, 33(65), pp. 99-108.

García Ramón, M. Dolors, Albet, Abel, Nogué, Joan Riudor, Lluis (1998): "Voices from the margins: gendered images of "otherness" in colonial Morocco", Gender, Place and Culture, 5(3), pp. 229-240.

García Ramón, M. Dolors \& Caballé, Alba (1999): "Els estudis de gènere. Un balanc bibliomètric internacional" IN Professor Joan Vilà Valentí. El seu mestratge en la geografia universitària, Barcelona: Universitat de Barcelona, Col.lecció Homenatges, pp. 333-345.
García Ramón, M. Dolors \& Nogué, Joan (1999): "Colonialismo, imperialismo $y$ exploración en geografía. Nuevas aportación críticas sobre orientalismo y postcolonialismo" IN Nogué, Joan \& Villanueva, José Luis (eds) España en Marruecos: Discursos geográficos e intervención terri torial, Lleida: Milenio, pp. 35-54.

García Ramón, M. Dolors \& Baylina, Mireia (eds.) (2000): El nuevo papel de las mujeres en el desarrollo mral, Barcelona: Oikos - Tau.

García Ramón, M. Dolors \& Baylina, Mireia (2001): "De la invisibilitat a l'agricultura ecològica: dona i gènere als estudis rurals", Treballs de la Societat Catalana de Geografia, 51, pp. 27-55.

García Ramón, M. Dolors (2003): "Gender and the colonial encounter in the Arab world:examining women's experiences and narratives", Environment and Planning D. Society and Space, 21, pp. 653672 .

García Ramón, M. Dolors (2004): "On diversity and difference in Geography: a Southern European perspective", European Urban and Regional Studies, 11(4), pp. 395-398.

García Ramón, M. Dolors \& Pujol, Herminia (2005): "La presencia de mujeres en la geografía académica: ¿Hacia una masculinización de la disciplina?", Cuademos de Geografia (en prensa)

Hancock, Claire (2002): "Genre et géographie: les apports des géographies de langue anglaisen, Espace, Populations, Societés, pp. 257-264.

Haraway, D. (1995): "Conocimientos situados: la cuestión científica en el feminismo y el privilegio de la persepctiva parcial",IN: Haraway, D. Ciencia, cyborgs y mujeres. La reinvención de la naturaleza, Valencia: Catedra, pp. 313-346. 
Hubbard, Phil (2000): "Desire/disgust:moral geographies of heterosexuality", Progress in Human Geography, 24, pp. 191-217.

Jackson, Peter (1991): "The cultural politics of masculinity: towards a social geography", Transactions of the Institute of British Geographers, 16(2), pp. 199-213.

Johnston, R. J. \& Sideway, J. (2004): Geography and geographers: Angloamerican buman geograpby since 1945, Londres: Arnold (sexta edición revisada).

Johnston, Lynda (1996): "Pumped-uo politics: female body builders refiguring the body" Gender, Place and Culture, 3(3), pp. 327340.

Johnston, Lynda (2001): "(Other) bodies and tourism studies", Annals of Tourism Research, 28(1), pp. 180-201.

Johnston, Lynda (2004): "Mobilising Pride: lesbian, tourism and parades", Comuni.

Jones III, J. P., Nast, H. J. \& Roberts, S. M. (eds) (1997): Thresholds in feminist geography: difference, metbodology, representation Oxford: Rowman \& Littlefield.

Katz, Cindi (1994): "Playing in the field", Professional Geographer, 46(1), pp. 67-72 (traducido al castellano en Perla Zusman, ed. Género, Espacio y Sociedad, Dossier de Lecturas, Curso de Tercer Ciclo de Doctorado en Geografía Humana, 2005).

Kitchin, Rob \& Lysaght, K. (2003): "Heterosexism and geographies of everyday life in Belfast", Environment and Planning A, 35, pp. 489-510.

Knopp, Lawrence (1990): Some theoretical implications of gay involvement in an urban land market" Political Geography Quarterly, 9, pp. 337-52.

Kwan, Mei-Po (2002): Dossier sobre "Feminist geography and GIS", Gender, Place and Culture, 9(3), pp. 261-303.
Little, Jo et al. (eds) (1988): Women in cities: Geography and gender in the urban environment, Basingstoke: MacMillan.

Little, Jo (2002): "Rural geography: rural gedber identity and the performance of masculinity and femininity in the countryside", Progress in Human Geograpby, pp. 665-670.

Longhurst, Robyn (1997): "(Dis)embodied geographies", Progress in Human Geograpby, 21(4), pp. 486-501.

Longhurst, Robyn (2001): Bodies: exploring fluid boundaries, Londres: Routledge.

López, Rosa (2000): "La agricultura ecológica como una alternativa también para las mujeres", Actas del $X$ Coloquio de Geografía Rural en España, Lleida: Universidad de Lleida, pp. 490-498.

Massey, Doreen (1995): "Masculinity, dualism and high technology", Transactions of the Instituteof British Geography, 20, pp. 487499.

Massey, Doreen (2004): "Lugar, identidad y geografía de la responsabilidad en un mundo contemporáneo", Treballs de la Societat Catalana, 57, pp. 77-84.

McDowell, Linda (1992): "Doing gender: feminism, feminist and research methods in human geography", Transactions of the Insitute of British Geographers, 17, pp. 399-416 (traducido al castellano en Perla Zusman,ed. Género, Espacio y Sociedad, Dossier de Lecturas, Curso de Tercer Ciclo de Doctorado en Geografía Humana, 2005).

McDowell, Linda (1993): "Space, place and gender relations: Part II. Identity, difference, feminist geometries and geographies", Progress in Human Geography, 17(3), pp. 305-318.

McDowell, Lynda (1995): "Body work: heterosexulal gender performances in city workplaces" IN Bell, David \& Valentine, 
Gill (eds) Mapping desire:geograpbies of sexualities, Londres: Routledge.

McDowell, Lynda (1999): Gender, identity and place:undestanding feminist geographies Cambridge: Polity Press (traducido al castellano como Género, identidad y lugar, Valencia: Cátedra, 2000).

McDowell, Linda (2003): Redundant masculinities?: Employment change and white working class youtb, Londres: Blackwell.

McLafferty, Sara (2005): "Geographic information and women's empowement: a breast cancer example", IN Nelson,Lise \& Seager,Joni (2005) A companion to Feminist Geograpby, Oxford: Blacwell, pp. 305-321.

Miraftab, Faranak (2004): "Can you belly dance? Methodological questions in the era of transnational feminist research", Gender, Place and Culture, 11(4) pp. 595604.

Monk, Janice (1996): "El lloc compta. Perspectives internacionals comparades sobre la geografia feminista", Documents d'Anàlisi Geogràfica, 26, pp. 245-256.

Nieto, Cristina (2004): Las empresarias rurales malagueñas. La respuesta de las mujeres al mercado laboral. Publicaciones de la Universidad de Málaga.

Nelson, Lise \& Seager, Joni (2005): A companion to Feminist Geograpby, Oxford: Blacwell.

Nogué, Joan, Albet, Abel, García Ramón, M. Dolors, Riudor, LLuis (1996): "Orientalisme, colonialisme i génere: el Marroc sensual i fanàtic d'Aurora Bertrana", Documents d'Anàlisi Geogràfica, 29, pp. 4-34.

Nogué, Joan, Zusman, Perla, García Ramón, M. D. (2005): Una mirada a l'África: viatgers $i$ viatgeres dels segles XIX $i$ XX, Barcelona:Proa (en prensa).
Ortega Valcárcel, J. (2000): Los borizontes de la geografía, Barcelona: Ariel.

Ortiz, Ana \& García Ramón, M. Dolors (2000): "The fixed term contract, tha Spanish route to flexibility? Women in the retail sector in the Barcelona region", Economic and Industrial Democracy: an International Joumal, 21(3) pp. 311-333.

Ortiz, Anna (2004): "Sens de lieu pour les femmes d'un quartier multiculturel de Barcelone (El Raval)", Espace, Populations et Societés, 1, pp. 59-69.

Ortiz, Anna, Albet, Abel, García Ramón, M. Dolors, Prats, María (2002): Urban planning, gender and social integration in Barcelona, Informe Final, WolkswagenStiftung, Universidad de Hanover (mimeo).

Pallarés, Montserrat; Tulla, Antoni F. PallarèsBlanch, Marta (2004): Capital social i treballs de les dones als Pirineus. El cas de l'Alt Pirineu. Barcelona: Institut Català de la Dona.

Panelli, R. (2004): Social Geograpbies: from Difference to Action, Londres: Sage.

Paravicini, Ursula (1990): Habitat ou fèminin, Lausanne: Presses Polytechniques et Universitaires Romanches.

Paravicini, Ursula \& Zempel-Gino, Maren (eds) (2003): Dokumentation. Wissenschaftliche Kolloquien 1999-2002, Hanover: NNFG.

Peake, Linda. \& Valentine, Gill (2003): "Editorial", Gender, Place and Culture, 10(2), pp. 107-109.

Pedone, Claudia (2000): "El trabajo de campo y los métodos cualitativos. Necesidad de nuevas reflexiones desde la geografía latinoamericana", Scripta Nova. Revista Electrónica de Geografía y Ciencias Sociales, 57, 1 febrero.

Pedone, Claudia (2003): "Las relaciones de género en la familia ecuatoriana dentro 
del contexto migratorio internacional hacia el estado español", Treballs de la Societat Catalana de Geografia, 56, pp. 79-106.

Prados , M. José (2000): Situación socioeconómica de las mujeres murales en España, Sevilla: Consejería de Agricultura, Junta de Andalucía.

Pratt, G. (1992): "Feminist Geography", Urban Geography, 13(4), pp. 385-391.

Cloke, P. Crang,Ph. \& Goodwin, M. (eds): Envisioning buman geographies, Londres: Arnold, pp. 128-145.

Prats, María, García Ramón, M. Dolors, Canoves, Gemma (1995): Las mujeres y el uso del tiempo, Madrid: Ministerio de Asuntos Sociales, Instituto de la Mujer.

Prats, María (1998): "Geografia feminista i metodologia: reflexió sobre un procés d'aprenentatge paral.lel", Cuadernos de Geografia, 64, pp. 313-323.

Prats, María \& García Ramón, M. Dolors (2004): "Emploi du temps et vie quotidienne des femmes adultes à Barcelonne. Espace, Population et Societés, 1, pp. 59-69.

Professional Geographer (1994): Dossier sobre "Women in the field", 46, pp. 54-102.

Professional Geographer (1995): Dossier sobre "Should women count? The role of quantitative methods in feminist research", 47, pp. 426-466.

Professional Geographer (2001): Dossier sobre "Women in geography in the $21^{\text {st}}$. century", 54, pp. 697-738.

Pujol, Herminia (2003) \& García Ramon, M. Dolors, (2003): "Female representation in academic geography in Spain: towards a masculinization of the discipline?, Journal of Geography and Higher Education, 28(1), pp. 111-119.

Riudor, Lluís (2005): "De la Rambla al Nil: els viatgers catalans (1859-1936)", Treballs de la Societat Catalana de Geografia, 58 (en prensa).

Romero, J et al. (coord.) (2004): Geografia Humana: procesos, riesgos e incertidumbres en un mundo globalizado, Barcelona: Ariel.

Rose, Gillian (1994): Feminism and geograpby: the limits of geograpbical knowledge, Cambridge: Polity.

Rose, Gillian (1997): "Situating knowledge: positionality, reflexivities and other tactics", Progress in Human Geography, 21(3), pp. 305-320.

Rose, Gillian (1995): "Geography and gender, cartographies and corporealities", Progress in Human Geography, 19(4), pp. $544-548$.

Sabaté, Ana \& Tulla, Antoni (1992): "Geografía y género en España: una aproximación a la situación actual" en Bosque Maurel J. et al. La geografia en España (1970-1990), Madrid: Fundación BBV, pp. $277-283$.

Sabaté, Ana (1993): "Industria rural en Toledo: la incorporación de las mujeres al mercado de trabajo", Anales de Geografia de la Universidad Complutense, 12, pp. 277-288.

Sabaté, A., Rodríguez, J. M. \& Díaz, M. A. (1995): Mujeres, espacio y sociedad:bacia una geografía del género, Madrid: Síntesis.

Sabaté, Ana (2000): "Género, medio ambiente y acción política: un debate pendiente en la geografía actual", Anales de Geografia de la Universidad Complutense, 20, pp. 177-191.

Sabaté, Ana (2002): "Rural development is getting female: old and new alternatives for women in rural areas in Spain", Antipode, 34(5), pp. 1.004-1.006.

Santos, Xosé M. (2002): "Espacios disidentes en los procesos de ordenación territorial", 
Documents d'Anàlisi Geogràfica, 40, pp. 69-104.

Silva, da Susana Veleda (2000): "Os etudos de gênero no Brasil: algunas consideraçoes" Biblio 3W. Revista Bibliografica de Geografia y Ciencias Sociales, 262, nov.

Solsona, Montserrat (1989): "El problema de la medición del trabajo de la mujer", Documents d'Anàlisi Geogràfica, 14, pp. 149-169.

Solsona, Montserrat \& Treviño, Rocío (1995): "Activitat, maternitat i paternitat a l'Europa comunitària", Documents d'Anàlisi Geogràfica, 26, pp. 191-207.

Solsona, Montserrat \& treviño, Rocío (2005): "Divorci i trajectòries post-ruptura a Catalunya", II Jornades de Població. La Població a Catalunya, Universidad Autónoma de Barcelona, CED (en prensa).

Townsend, Janet (1995): "Es pot parlar en nom dels altres?" Documents d'Anàlisi Geogrăfica, 26, pp. 209-218.
Unión Geográfica Internacional (UGI, International Geographical Union) (2004): One Eartb, Many Worlds, 30 Congreso Internacional de Geografía, Glasgow (mimeo)

Valentine, Gill (2001): Social Geographies. Space and Society, Harlow: Prentice Hall.

Valentine, Gill (2002): "People like us: negotiating sameness and difference in the research process" IN Moss,Pamela, Feminist Geography in Practice: Research and Methods, Oxford: Blackwell, pp. 116 126.

Women and Geography Study Group (Royal Geographical Society and IBG) (1997): Feminist Geographies. Explorations in Diversity and Difference, Essex: Longman.

Women and Geography Study Group (Royal Geographical Society and IBG) (2004): Geograpby and Gender Reconsidered, en formato de $\mathrm{CD}$. 